\title{
Identification of a Population of X-Ray-Emitting Massive Stars in the Galactic Plane
}

\section{Citation}

Anderson, Gemma E., B. M. Gaensler, David L. Kaplan, Bettina Posselt, Patrick O. Slane, Stephen S. Murray, Jon C. Mauerhan, et al. 2011. "IDENTIFICATION OF A POPULATION OF XRAY-EMITTING MASSIVE STARS IN THE GALACTIC PLANE." The Astrophysical Journal 727 (2) (January 11): 105. doi:10.1088/0004-637x/727/2/105.

\section{Published Version}

doi:10.1088/0004-637X/727/2/105

\section{Permanent link}

http://nrs.harvard.edu/urn-3:HUL.InstRepos:14068420

\section{Terms of Use}

This article was downloaded from Harvard University's DASH repository, and is made available under the terms and conditions applicable to Open Access Policy Articles, as set forth at http:// nrs.harvard.edu/urn-3:HUL.InstRepos:dash.current.terms-of-use\#OAP

\section{Share Your Story}

The Harvard community has made this article openly available.

Please share how this access benefits you. Submit a story.

\section{Accessibility}




\title{
IDENTIFICATION OF A POPULATION OF X-RAY-EMITTING MASSIVE STARS IN THE GALACTIC PLANE
}

\author{
Gemma E. Anderson ${ }^{1}$, B. M. Gaensler ${ }^{1,12}$, David L. Kaplan ${ }^{2,13,14}$, Bettina Posselt ${ }^{3}$, Patrick O. Slane ${ }^{3}$, \\ Stephen S. Murray ${ }^{3}$, Jon C. Mauerhan ${ }^{4}$, Robert A. Benjamin ${ }^{5}$, Crystal L. Brogan ${ }^{6}$, \\ Deepto Chakrabarty ${ }^{7}$, Jeremy J. Drake ${ }^{3}$, Janet E. Drew ${ }^{8}$, Jonathan E. Grindlay ${ }^{3}$, Jaesub Hong ${ }^{3}$, \\ T. Joseph W. Lazio ${ }^{9}$, Julia C. LeE ${ }^{3}$, Danny T. H. Steeghs ${ }^{10}$, and Marten H. van KerkwiJK ${ }^{11}$ \\ ${ }^{1}$ Sydney Institute for Astronomy, School of Physics A29, The University of Sydney, NSW 2006, Australia; g.anderson@ physics.usyd.edu.au \\ ${ }^{2}$ Kavli Institute for Theoretical Physics, Kohn Hall, University of California, Santa Barbara, CA 93106, USA \\ ${ }^{3}$ Harvard-Smithsonian Center for Astrophysics, Cambridge, MA 02138, USA \\ ${ }^{4}$ Spitzer Science Center, California Institute of Technology, Pasadena, CA 91125, USA \\ ${ }^{5}$ Department of Physics, University of Wisconsin, Whitewater, WI 53190, USA \\ ${ }^{6}$ National Radio Astronomy Observatory, Charlottesville, VA 22903, USA \\ ${ }^{7}$ MIT Kavli Institute for Astrophysics and Space Research and Department of Physics, Massachusetts Institute of Technology, Cambridge, MA 02139, USA \\ ${ }^{8}$ Centre for Astrophysics Research, STRI, University of Hertfordshire, Hatfield AL10 9AB, UK \\ ${ }^{9}$ Naval Research Laboratory, Washington, DC 20375, USA \\ ${ }^{10}$ Department of Physics, University of Warwick, Coventry CV4 7AL, UK \\ ${ }^{11}$ Department of Astronomy and Astrophysics, University of Toronto, Toronto, ON M5S 3H4, Canada \\ Received 2010 August 31; accepted 2010 November 11; published 2011 January 11
}

\begin{abstract}
We present X-ray, infrared, optical, and radio observations of four previously unidentified Galactic plane X-ray sources: AX J163252-4746, AX J184738-0156, AX J144701-5919, and AX J144547-5931. Detection of each source with the Chandra X-ray Observatory has provided sub-arcsecond localizations, which we use to identify bright infrared counterparts to all four objects. Infrared and optical spectroscopy of these counterparts demonstrate that all four X-ray sources are extremely massive stars, with spectral classifications: Ofpe/WN9 (AX J163252-4746), WN7 (AX J184738-0156 = WR121a), WN7-8h (AX J144701-5919), and OIf ${ }^{+}$(AX J144547-5931). AX J163252-4746 and AX J184738-0156 are both luminous, hard, X-ray emitters with strong Fe XXV emission lines in their X-ray spectra at $\sim 6.7 \mathrm{keV}$. The multi-wavelength properties of AX J163252-4746 and AX J184738-0156 are not consistent with isolated massive stars or accretion onto a compact companion; we conclude that their X-ray emission is most likely generated in a colliding-wind binary (CWB) system. For both AX J144701-5919 and AX J144547-5931, the X-ray emission is an order of magnitude less luminous and with a softer spectrum. These properties are consistent with a CWB interpretation for these two sources also, but other mechanisms for the generation of X-rays cannot be excluded. There are many other as yet unidentified X-ray sources in the Galactic plane, with X-ray properties similar to those seen for AX J163252-4746, AX J184738-0156, AX J144701-5919, and AX J144547-5931. This may indicate a substantial population of X-ray-emitting massive stars and CWBs in the Milky Way.
\end{abstract}

Key words: stars: winds, outflows - stars: Wolf-Rayet - supergiants - X-rays: binaries - X-rays: individual (AX J163252-4746, AX J184738-0156, AX J144701 -5919, AX J144547-5931) - X-rays: stars

Online-only material: color figure

\section{INTRODUCTION}

Wolf-Rayet (WR) stars and their O-type supergiant progenitors (Of) evolve from the most massive stars in our Galaxy, with initial masses $\gtrsim 25 M_{\odot}$. These evolved stars, particularly WR, have extremely strong stellar winds, experiencing high mass-loss rates of $\dot{M} \sim 10^{-5} M_{\odot} \mathrm{yr}^{-1}$ and, in some cases, have luminosities $>10^{6} L_{\odot}$ (Crowther 2008). Their short lifetimes make them very rare; $<400$ WR stars are known in our Galaxy (van der Hucht 2006; Martins et al. 2008; Shara et al. 2009; Mauerhan et al. 2009, 2010) and are usually only found in the Galactic plane.

Massive stars have historically been discovered through optical and infrared observations and are classified based on their spectral characteristics in these wavebands. However, $\mathrm{X}$-ray observations are now becoming a newly recognized technique for discovering massive stars and are also a powerful

\footnotetext{
${ }^{12}$ Australian Research Council Federation Fellow.

${ }^{13}$ Hubble Fellow.

${ }^{14}$ Current address: Department of Physics, University of Wisconsin, Milwaukee, WI 53201-0431, USA.
}

tool in assessing their physical environments (e.g., Mauerhan et al. 2010), allowing us to determine if they are isolated or in a high-mass X-ray binary (HMXB) or colliding-wind binary (CWB) system. By discovering more of these massive stars and understanding their emission mechanisms, we can determine how mass-loss drives the different stages of stellar evolution.

The most accepted model for X-ray generation in a single hot star is the instability-driven wind-shock picture, which attributes the production of soft, thermal X-ray emission, with temperatures of $k T<1 \mathrm{keV}$, to shocks distributed throughout the wind (Lucy \& White 1980; Lucy 1982). More exotic models of X-ray generation in isolated massive stars include the magnetically channeled wind-shock mechanism, which was first explored in detail by Babel \& Montmerle (1997a, 1997b). In this case a radiatively driven stellar wind is magnetically channeled from the two hemispheres of the star. These streams collide at the magnetic equator and the rapid deceleration causes shock heating resulting in high levels of hard thermal X-ray emission. There is also the possibility of isolated OB supergiant stars producing intrinsic hard non-thermal $\mathrm{X}$-rays through inverse Compton scattering (Chen \& White 1991). 
Alternatively, the X-ray emission may not be completely intrinsic to the massive star but created through a binary interaction. X-rays can be generated in HMXBs through gravitational accretion onto a compact object such as a neutron star (NS) or black hole $(\mathrm{BH})$. The two main classes of HMXBs are the Be $\mathrm{X}$-ray binary systems (BeXs) and the supergiant X-ray binaries (SGXBs). BeXs are accretion fed by a disk around a Be star and are often transient X-ray sources whereas SGXBs are wind-fed, and persistent, X-ray sources (McClintock \& Remillard 2006).

CWB systems are another class of massive stellar binaries marked by extreme wind loss and high-energy emission. Originally predicted by Prilutskii \& Usov (1976) and Cherepashchuk (1976), the supersonic winds from the two massive stars in a binary produce shock-heated gas (Stevens et al. 1992), resulting in hard, thermal X-ray emission (Pittard \& Parkin 2010) and possibly $\gamma$-rays, likely produced by inverse Compton scattering (e.g., Benaglia \& Romero 2003; Pittard \& Dougherty 2006; Reimer et al. 2006; De Becker 2007). The detection of this highenergy emission allows us to probe the nature of these shocks and provides a laboratory for investigating particle acceleration in a very different density regime to that in supernova remnants.

In this paper we discuss our classification of four previously unidentified Galactic X-ray sources: AX J163252-4746, AX J184738-0156, AX J144701-5919, and AX J144547-5931. These sources have been observed with the Chandra X-ray Observatory as part of the "ChIcAGO" (Chasing the Identification of ASCA Galactic Objects) project (G. E. Anderson et al. 2011, in preparation), a survey designed to localize and classify the unidentified X-ray sources discovered during the ASCA Galactic Plane Survey (AGPS; Sugizaki et al. 2001). The Advanced Satellite for Cosmology and Astrophysics (ASCA) surveyed the inner region of the Galactic plane, detecting 163 $\mathrm{X}$-ray sources with fluxes between $10^{-11}$ and $10^{-13} \mathrm{erg} \mathrm{cm}^{-2} \mathrm{~s}^{-1}$ in the $0.7-10.0 \mathrm{keV}$ energy range. Due to the $A S C A \mathrm{X}$-ray telescope's $\sim 3^{\prime}$ spatial resolution, only a third of these X-ray sources have been properly characterized and little is known about the remaining unidentified objects. This unidentified population should contain at least some rare classes of X-ray objects, as modeling by Hands et al. (2004) and Grindlay et al. (2005) has demonstrated that the Galactic populations of cataclysmic variables, bright $\mathrm{X}$-ray binaries, and background active galactic nuclei cannot account for the entire observed flux distribution of $\mathrm{X}$-ray sources detected in the AGPS. In ChIcAGO, we are combining the sub-arcsecond localization capabilities of Chandra with a detailed multi-wavelength follow-up program, with the goal of classifying the $>100$ unidentified sources in the AGPS.

We chose to begin our investigation with AX J163252-4746, AX J184738-0156, AX J144701-5919, and AX J144547-5931 (listed in order of decreasing X-ray flux) as they are all highly absorbed with bright infrared counterparts (8 $\mu \mathrm{m}$ magnitude $<7$ ), making them distant, luminous and unusual objects. The Chandra observations and follow-up studies are described in Section 2, along with the results from these observations. In Section 3 we discuss the X-ray spectra of these sources and classification of their counterparts, demonstrating that these four objects are all X-ray-emitting massive stars.

\section{OBSERVATIONS AND RESULTS}

\subsection{Chandra and Archival XMM-Newton Data}

Our Chandra observations of AX J163252-4746, AX J184738-0156, AX J144701-5919, and AX J144547-5931 were short (between 1 and $3 \mathrm{ks}$ per source). This allowed us to detect $\sim 100$ counts per target so as to fulfill the primary aim to localize each source, while providing limited spectral information. We observed AX J184738-0156, AX J144701-5919, and AX J144547-5931 with the Advanced CCD Imaging Spectrometer (ACIS; Garmire et al. 2003) in the ACIS-S mode. As AX J163252-4746 has a predicted ACIS count-rate $>0.2$ counts $\mathrm{s}^{-1}$, we used the High Resolution Camera (HRC; Murray 2000) in the HRC-I mode in order to avoid the positional degradation associated with pile-up (Davis 2001) that would arise from an ACIS observation.

These data were reduced using the Chandra Interactive Analysis of Observation software (CIAO), version 4.1, following the standard reduction recipes given in the online CIAO 4.1 Science Threads. ${ }^{15}$ Further details on the Chandra observations will be published by G. E. Anderson et al. (2011, in preparation). For each of the four AGPS targets, a Chandra source with $50-150$ counts was detected within $3^{\prime}$ of the published ASCA position. The need for Chandra observations to localize the AGPS sources is illustrated in Figure 1, where each Chandra detection clearly shows a much more precise source position (white contour) when compared to the original ASCA detection (black contours). The Chandra instruments and exposure times used for the observations can be found in Table 1. The resulting Chandra position for each source is listed in Table 2.

AX J163252-4746 and AX J184738-0156 were also detected off-axis in archival XMM-Newton data (observational IDs 0201700301 and 0203850101 , respectively), with $>9600$ counts each. These two sources are catalogued under the names 2XMM J163248.6-474504 and 2XMM J184736.6-015633, respectively, in the XMM-Newton Serendipitous Source catalogue (2XMMi; Watson et al. 2009). The details on these observations can also be found in Table 1. (AX J163252-4746 was also detected off-axis in ten other XMM observations. However, we chose to concentrate our analysis on observational ID 0201700301 as it has the longest integration and therefore the greatest number of counts detected.)

We chose to model the XMM spectra of AX J163252-4746 and AX J184738-0156, rather than their Chandra data, as the HRC instrument does not provide any spectral information for AX J163252-4746 and the XMM observations detected many more source counts for AX J184738-0156. We fit the XMM spectra of AX J163252-4746 and AX J184738-0156 with absorbed Raymond \& Smith (1977) models as both sources show a strong emission line between 6 and $7 \mathrm{keV}$, indicating there is likely a thermal component to the X-ray emission. The $X M M$ spectra of both AX J163252-4746 and AX J184738-0156 are shown in Figure 2. ${ }^{16}$ Using a Gaussian profile to model the emission line in the $X M M$ spectra, the line was found to be unresolved for both AX J163252-4746 and AX J184738-0156. In each case the equivalent width $(\mathrm{EW})$ is $\sim 1.3_{-0.2}^{+0.1} \mathrm{keV}$, at energies $6.67 \pm 0.01$ and $6.66 \pm 0.01 \mathrm{keV}$ (90\% confidence) for AX J163252-4746 and AX J184738-0156, respectively, where the $X M M$ energy resolution is FWHM $\sim 135-140 \mathrm{eV}$ around $7 \mathrm{keV}{ }^{17}$ We identify both emission lines as Fe XXV, which has an approximate energy of $6.7 \mathrm{keV}$. The absorbed

\footnotetext{
15 http://cxc.harvard.edu/ciao/threads/

16 The pipeline-produced archival XMM spectra were fit using XSPEC (Dorman \& Arnaud 2001). We note that in the XMM observation of AX J163252-4645, many scatter arcs from the nearby bright X-ray binary 4U 1630-47 are present. This could lead to poor background subtraction in the $X M M$ reduction pipeline. However, the other XMM archival observations of AX J163252-4746, which do not have scatter arcs, show very similar spectral shapes.

17 http://www.mssl.ucl.ac.uk/www_xmm/ukos/onlines/uhb/XMM_UHB/ node28.html
} 

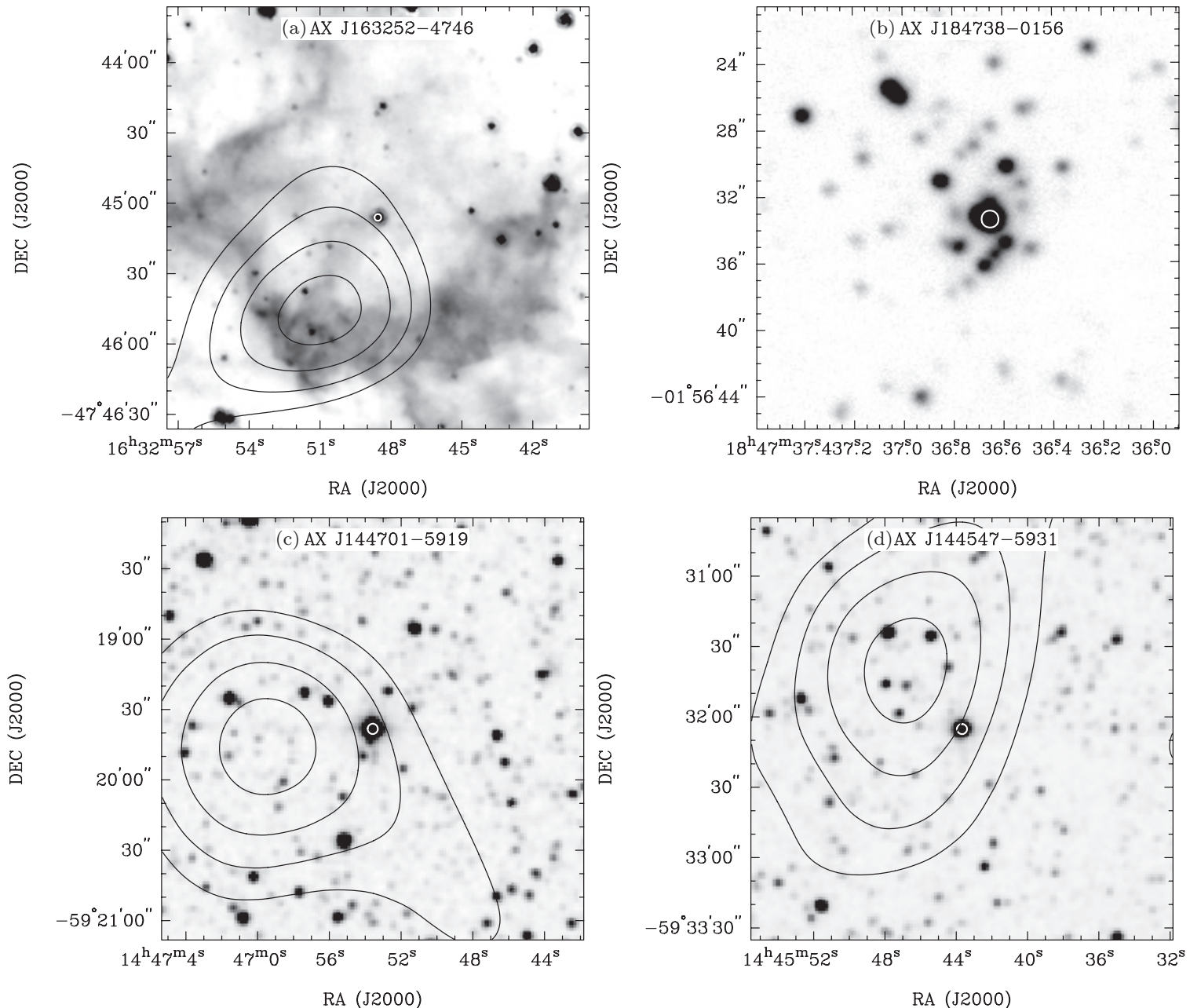

Figure 1. Infrared images of the regions surrounding AX J163252-4746, AX J184738-0156, AX J144701-5919, and AX J144547-5931. The white contours represent the smoothed Chandra detection of AX J163252-4746 and AX J184738-0156 (each at 95\% of the peak count-rate), and AX J144701-5919 and AX J144547-5931 (each at $40 \%$ of the peak count-rate). In each case the infrared counterpart to the X-ray source is clearly detected. The black contours show the original ASCA detection, in the $0.7-10.0 \mathrm{keV}$ energy range, of each source at $65 \%, 75 \%, 85 \%$, and $95 \%$ of the peak count-rate. (a) GLIMPSE $8 \mu$ m image of AX J163252-4746. (b) PANIC $H$-band image of the star cluster in which AX J184738-0156 is embedded. The Chandra contour is clearly coincident with the brightest member of the cluster. The field of view of this image is too small to overlay the ASCA contours. (c) 2MASS $K$-band image of AX J144701-5919. (d) 2MASS $K$-band image of AX J144547-5919.

Table 1

X-ray Observations of AX J163252-4746, AX J184738-0156, AX J144701-5919, and AX J144547-5931

\begin{tabular}{ccccccc}
\hline \hline Source & X-ray Telescope & ObsID & Date (yyyy-mm-dd) & Instrument & Exp Time (s) & Total Counts \\
\hline AX J163252-4746 & Chandra & 8155 & $2007-10-23$ & HRC-I & 2670 & $68 \pm 8$ \\
& $X M M$ & 0201700301 & $2004-08-19$ & EPIC MOS1 & 50987 & $3539 \pm 65$ \\
& & & EPIC MOS2 & 51081 & $3304 \pm 62$ \\
& & & EPIC PN & 46742 & $7338 \pm 94$ \\
AX J184738-0156 & Chandra & 9612 & $2008-05-27$ & ACIS-S & 1650 & $123 \pm 11$ \\
& XMM & 0203850101 & $2004-10-22$ & EPIC MOS1 & 25131 & $2425 \pm 54$ \\
& & & & EPIC MOS2 & 25155 & $2311 \pm 52$ \\
AX J144701-5919 & Chandra & 8233 & EPIC PN & 20632 & $4939 \pm 77$ \\
AX J144547-5931 & Chandra & 8240 & $2007-01-13$ & ACIS-S & 1450 & $83 \pm 9$ \\
& & & $2007-08-12$ & ACIS-S & 2550 & $69 \pm 8$ \\
\hline
\end{tabular}

Note. ${ }^{a}$ Total number of counts in the $0.3-8.0 \mathrm{keV}$ energy range for ACIS-S, the full Chandra energy range (0.1-10.0 keV) for HRC-I and 0.2-12.0 $\mathrm{keV}$ energy range for $X M M$.

Raymond-Smith modeling of both AX J163252-4746 and AX J184738-0156 includes smaller bumps in the spectral fits, as seen in Figure 2, which could indicate the presence of other spectral lines. However, the error bars in the data points are too large for the existence of other ionic species to be confirmed.
There is no evidence for short-term variability within our Chandra and archival XMM observations between $\sim 330 \mathrm{~s}$ and $\sim 51000 \mathrm{~s}, \sim 210 \mathrm{~s}$ and $\sim 25100 \mathrm{~s}, \sim 180 \mathrm{~s}$ and $\sim 1450 \mathrm{~s}$, and $\sim 320$ s and $\sim 2550$ s for AX J163252-4746, AX J184738-0156, AX J144701-5919, and AX J144547-5931, respectively. There 
Table 2

X-ray Positions and Spectral Modeling of AX J163252-4746, AX J184738-0156, AX J144701-5919, and AX J144547-5931

\begin{tabular}{|c|c|c|c|c|c|c|c|c|c|}
\hline \multirow{2}{*}{$\begin{array}{l}\text { Source } \\
\text { AX }\end{array}$} & \multicolumn{2}{|c|}{ Chandra Position $^{\mathrm{a}}$} & \multirow{2}{*}{$\begin{array}{c}\text { Telescope } \\
\text { ObsID }^{\mathrm{b}}\end{array}$} & \multirow{2}{*}{$\begin{array}{c}\text { Distance } \\
(\mathrm{kpc})\end{array}$} & \multicolumn{5}{|c|}{ Raymond-Smith Fit Parameters ${ }^{\mathrm{c}}$} \\
\hline & R.A.(J2000) & Decl.(J2000) & & & $\begin{array}{c}k T(\mathrm{keV}) \\
\chi_{\mathrm{red}}^{2} \\
\end{array}$ & $\begin{array}{c}N_{\mathrm{H}} \\
F_{x, \text { abs }}\end{array}$ & Abundances $^{\mathrm{d}}$ & $\begin{array}{l}F_{x, \text { unabs }} \\
L_{x, \text { unabs }}\end{array}$ & $\log \left(L_{x} / L_{\mathrm{bol}}\right)^{\mathrm{e}}$ \\
\hline J163252-4746 & $16: 32: 48.548$ & $-47: 45: 06.20$ & $\begin{array}{c}X M M \\
0201700301\end{array}$ & $\sim 4.5$ & $\begin{array}{c}3.2 \pm 0.2 \\
0.9\end{array}$ & $\begin{array}{l}6.2 \pm 0.3 \\
3.7 \pm 0.1\end{array}$ & $0.9 \pm 0.1$ & $\begin{array}{c}11.9_{-0.5}^{+0.6} \\
\sim 3.4\end{array}$ & $\sim-5.4--5.1$ \\
\hline J184738-0156 & $18: 47: 36.650$ & $-01: 56: 33.32$ & $\begin{array}{c}\text { XMM } \\
0203850101 \\
\text { Chandra } \\
9612\end{array}$ & $\sim 5.6$ & $\begin{array}{c}3.1 \pm 0.3 \\
0.8 \\
7.5(>1.9) \\
0.7\end{array}$ & $\begin{array}{c}8.9 \pm 0.5 \\
2.2 \pm 0.1 \\
10.5_{-5.0}^{+11.7} \\
2.0(>0.3)\end{array}$ & $\begin{array}{c}0.7 \pm 0.1 \\
0.7\end{array}$ & $\begin{array}{c}9.1_{-0.6}^{+0.9} \\
\sim 3.4\end{array}$ & $\sim-5.4--5.0$ \\
\hline J144701-5919 & $14: 46: 53.583$ & $-59: 19: 38.31$ & $\begin{array}{c}\text { Chandra } \\
8233\end{array}$ & $\sim 2.6$ & $\begin{array}{c}1.3_{-0.5}^{+1.3} \\
0.2\end{array}$ & $\begin{array}{l}2.9_{-1.3}^{+1.7} \\
0.5_{-0.4}^{+1.0}\end{array}$ & 1.0 & $\begin{array}{c}\sim 4 \\
\sim 0.32\end{array}$ & $\sim-6.4--6.0$ \\
\hline J144547-5931 & $14: 45: 43.673$ & $-59: 32: 05.25$ & $\begin{array}{c}\text { Chandra } \\
8240\end{array}$ & $\sim 2.3-3.0$ & $\begin{array}{c}1.2_{-0.8}^{+8.9} \\
0.3\end{array}$ & $\begin{array}{c}3.5_{-2.1}^{+4.5} \\
0.3(<1.6)\end{array}$ & 1.0 & $\begin{array}{c}\sim 3 \\
\sim 0.19-0.32\end{array}$ & $\sim-6.5--6.3$ \\
\hline
\end{tabular}

Notes. In the cases where one of the $90 \%$ errors for a given spectral fit parameter was incalculable we quote the best-fit parameter followed by a $90 \%$ confidence upper or lower limit. The $X M M$ spectra were used rather than the Chandra spectrum to calculate the unabsorbed flux and luminosity of AX J184738-0156 as the model fits to the XMM data are far more statistically significant.

a All position errors are within a $00^{\prime \prime} 8$ radius circle for a $95 \%$ confidence.

${ }^{\mathrm{b}} \mathrm{X}$-ray telescope and corresponding observation identification used in the spectral modeling.

c The best-fit Raymond-Smith model parameters including temperature $k T(\mathrm{keV})$, absorption column density $N_{\mathrm{H}}\left(10^{22} \mathrm{~cm}^{-2}\right)$, reduced chi-sqaure $\chi_{\text {red }}^{2}$ from Chi Gehrels statistics and abundances relative to the solar value given by Anders \& Grevesse (1989). The absorbed X-ray flux, $F_{x}$, is in the 0.3-8.0 keV and 0.4-10.0 keV energy range for the Chandra and XMM fits, respectively. The unabsorbed X-ray flux, $F_{x, \text { unab }}\left(10^{-12} \mathrm{erg} \mathrm{cm}^{-2} \mathrm{~s}^{-1}\right)$ and the unabsorbed X-ray luminosity, $L_{x}$, unab,$\left(10^{34} \mathrm{erg} \mathrm{s}^{-1}\right)$ are calculated from the Raymond-Smith fits over the $0.3-8.0 \mathrm{keV}$ energy range. All fit parameter errors are for $90 \%$ confidence.

d The abundance parameter was prevented from varying during the fitting process of the Chandra spectra. The parameter was set to the number listed, which is relative to the solar value given by Anders \& Grevesse (1989). The abundance parameter was allowed to vary during the fitting of the $X M M$ spectra.

${ }^{\mathrm{e}}$ A range of possible $\log \left(L_{x} / L_{\mathrm{bol}}\right)$ calculated using the bolometric luminosity, $L_{\mathrm{bol}}\left(\mathrm{erg} \mathrm{s}^{-1}\right)$, of sources with similar spectral types taken from Table 3 of Mauerhan et al. (2010) and Table 5 of Oskinova (2005). This ratio was calculated using WN7-8h stars for AX J184738-0156 as its $K$-band spectral morphology (see Figure 5 in Blum et al. 1999) is similar to stars of this subtype seen in Martins et al. (2008) and Mauerhan et al. (2010). In the case of AX J144547-5931 we used the value of $L_{\text {bol }}$ that Mauerhan et al. (2010) calculated for X-ray source CXOGC J174617.0-285131, which is classified as an $\mathrm{O} \mathrm{If}^{+}$star

is also little evidence for long-term variability as the derived fluxes from power-law fits to the Chandra and $X M M^{18}$ observations of each source are within a factor of two from the original $A S C A$ flux, also derived from a power-law fit, published by Sugizaki et al. (2001). Using the Chandra and XMM data sets we also determined that there is no evidence for periodicity between $\sim 0.02$ and $17000 \mathrm{~s}, \sim 0.15$ and $8400 \mathrm{~s}, \sim 6.4$ and $480 \mathrm{~s}$ and $\sim 6.4$ and $850 \mathrm{~s}$ in AX J163252-4746, AX J184738-0156, AX J144701-5919, and AX J144547-5931, respectively, for a $99.9 \%$ confidence.

In order to determine the validity of spectral fits applied to the Chandra spectra of AX J144701-5919 and AX J144547-5931 we compared the XMM and Chandra spectra of AX J184738-0156. We used the XMM best-fit absorbed Raymond-Smith values to fit the Chandra spectrum of AX J184738-0156, allowing the normalization parameter to vary. The resulting fit is shown in blue in Figure 2(b) demonstrating that the Chandra spectrum is compatible with the XMM fits. An independent absorbed Raymond-Smith fit to the Chan$d r a$ spectrum results in best-fit values that have very large uncertainties. It is therefore difficult to start from the low-statistics of the Chandra data and then constrain the parameters of the source's spectrum. We therefore limit our interpretation of the AX J144547-5931 and AX J144701-5919 Chandra spectra by using absorbed Raymond-Smith fits only to estimate the unabsorbed X-ray flux. ${ }^{19}$ With this caveat, the Raymond-Smith

\footnotetext{
18 We also included a Gaussian profile component to the power-law fit of the $X M M$ data in order to model the flux in the $6.7 \mathrm{keV} \mathrm{Fe} \mathrm{XXV} \mathrm{emission} \mathrm{line.}$

19 The Chandra spectra were fit using the CIAO spectral fitting tool Sherpa.
}

spectral fit parameters for all four sources are summarized in Table 2.

\subsection{Multi-wavelength Data}

\subsubsection{Survey and Catalog Comparisons}

Comparison of the Chandra positions of AX J163252-4746, AX J184738-0156, AX J144701-5919, and AX J144547-5931 to optical and infrared catalogs allow us to discover any multi-wavelength counterparts to these X-ray sources. AX J144701-5919 and AX J144547-5931 are both faintly detected at optical wavelengths (R magnitude $>15.8$ ) in the US Naval Observatory (USNO) B catalog, version 1.0 (Monet et al. 2003). In both the Two Micron All Sky Survey (2MASS; Skrutskie et al. 2006) and the Galactic Legacy Infrared Mid-Plane Survey Extraordinaire (GLIMPSE; Benjamin et al. 2003) all four AGPS sources are shown to have clear counterparts at near-infrared and mid-infrared wavelengths. The corresponding 2MASS and GLIMPSE source names and magnitudes are listed in Table 3.

The GLIMPSE data also show that AX J163252-4746 is surrounded by a diffuse shell of $8 \mu \mathrm{m}$ emission (see Figure 1(a)). This emission is likely due principally to polycyclic aromatic hydrocarbons (PAHs) excited by soft ultraviolet radiation, detected within the broadband $8 \mu \mathrm{m}$ filter (Watson et al. 2008). Thus, such shells are signposts for hot stars with powerful stellar winds that are impacting the interstellar medium (Churchwell et al. 2006, 2007).

Examination of the $24 \mu \mathrm{m}$ mosaic images from the MIPSGAL Survey (Carey et al. 2009) shows that AX J163252-4746, 
Table 3

Infrared Magnitudes and Radio Fluxes of Counterparts to AX J163252-4746, AX J184738-0156, AX J144701-5919, and AX J144547-5931

\begin{tabular}{|c|c|c|c|c|c|c|c|c|c|c|c|}
\hline \multirow{2}{*}{$\begin{array}{l}\text { Source } \\
\text { AX }\end{array}$} & \multicolumn{3}{|c|}{ 2MASS Magnitudes ${ }^{\mathrm{a}}$} & \multicolumn{4}{|c|}{ GLIMPSE Magnitudes $^{\mathrm{b}}$} & \multicolumn{4}{|c|}{ ATCA Fluxes $(\mathrm{mJy})^{\mathrm{c}}$} \\
\hline & \multicolumn{3}{|c|}{ Catalog Source Name } & \multicolumn{4}{|c|}{ Catalog Source Name } & $1.4 \mathrm{GHz}$ & $2.4 \mathrm{GHz}$ & $4.8 \mathrm{GHz}$ & $8.0 \mathrm{GHz}$ \\
\hline J163252-4746 & $13.36 \pm 0.04$ & $>10.89$ & $>9.27$ & $8.18 \pm 0.06$ & $7.43 \pm 0.06$ & $7.27 \pm 0.03$ & $6.76 \pm 0.03$ & $<7.0$ & $<3.0$ & $2.6 \pm 0.2$ & $2.9 \pm 0.3$ \\
\hline J184738-0156 & $\begin{array}{r}2 \mathrm{MAs} \\
14.85 \pm 0.07 \\
2 \mathrm{MAs}\end{array}$ & $\begin{array}{c}\mathrm{J} 16324851-4 \\
11.01 \pm 0.06 \\
\mathrm{~J} 18473666-0\end{array}$ & $\begin{array}{l}5062 \\
8.66 \pm 0.04 \\
6334\end{array}$ & & $\begin{array}{l}\text { SSTGLMC G3 } \\
\text { SSTGLMA G }\end{array}$ & $\begin{array}{l}5085+00.1571 \\
5.48 \pm 0.19^{*} \\
7667-00.0346\end{array}$ & & & & & \\
\hline J144701-5919 & $8.93 \pm 0.02$ & $7.60 \pm 0.04$ & $6.82 \pm 0.02$ & $6.70 \pm 0.09^{*}$ & $\begin{array}{r}6.33 \pm 0.09^{*} \\
\end{array}$ & $5.57 \pm 0.03$ & $5.32 \pm 0.03$ & $27.0 \pm 0.6$ & $23.4 \pm 0.5$ & $16.8 \pm 0.2$ & $9.9 \pm 0.2$ \\
\hline J144547-5931 & $\begin{array}{c}9.23 \pm 0.04 \\
2 \mathrm{MAS}\end{array}$ & $\begin{array}{c}8.10 \pm 0.03 \\
\mathrm{~J} 14454369-5\end{array}$ & $\begin{array}{l}7.52 \pm 0.02 \\
2050\end{array}$ & $7.07 \pm 0.05$ & $\begin{array}{r}6.89 \pm 0.04 \\
\text { SSTGLMC G3 }\end{array}$ & $\begin{array}{c}6.71 \pm 0.03 \\
9656+00.1864\end{array}$ & $6.63 \pm 0.02$ & & & & \\
\hline
\end{tabular}

Notes. AX J184738-0156 was detected in the GLIMPSE 3.6, 4.5, and $8.0 \mu \mathrm{m}$ bands but no magnitudes were obtained as the source was either saturated or the surrounding region too confused.

a $J, H$, and $K_{s}$ counterpart magnitudes are taken from the 2MASS All-Sky Catalog of Point Sources (Skrutskie et al. 2006). Due to blending with nearby sources only 95\% confidence lower limits are quoted for the $H$ and $K$ magnitudes of AX J163252-4746.

${ }^{\mathrm{b}} m_{3.6}-m_{8.0}$ refers to the counterpart magnitudes at 3.6-8.0 $\mu \mathrm{m}$ taken from the GLIMPSE I DR5 Catalog. Magnitudes labeled with * are from the GLIMPSE I DR5 Archive, which is described as more complete but less reliable than the GLIMPSE I DR5 Catalog.

${ }^{c}$ ATCA radio observations were only obtained for AX J144701-5919 and AX J163252-4746. The $1 \sigma$ flux errors are quoted. No radio counterpart to AX J163252-4746 was detected at 1.4 and $2.4 \mathrm{GHz}$ so $5 \sigma$ flux upper limits are quoted. 

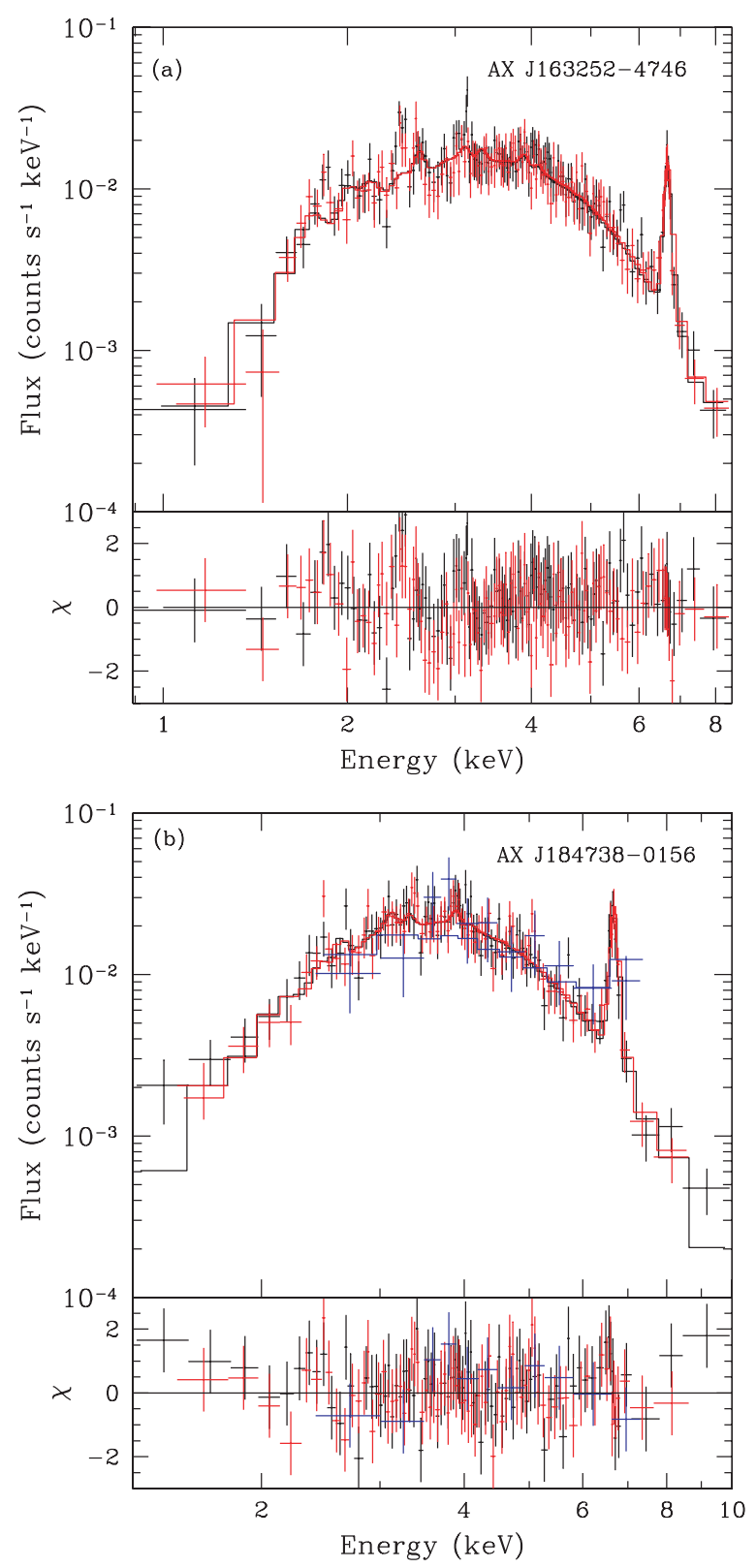

Figure 2. X-ray spectra of AX J163252-4746 and AX J184738-0156. The $X M M$ EPIC-MOS1 (black) and EPIC-MOS2 (red) spectra of these sources (data points) are overlaid with an absorbed Raymond-Smith fit (solid lines). The bottom panel in both (a) and (b) shows the residuals of the best-fit Raymond-Smith plasma for each detector. (a) XMM spectra of AX J163252-4746. (b) XMM spectra of AX J184738-0156 overlaid with the Chandra (blue) spectrum of this source. The solid blue line is the Chandra spectral fit using the best-fit parameters from the absorbed Raymond-Smith fit to the XMM data but allowing the normalization to vary. The Chandra residuals from this fit are also seen in the bottom panel.

(A color version of this figure is available in the online journal.)

AX J144701-5919, and AX J144547-5931 are also detected at this wavelength. AX J184738-0156 is situated within the H II region W43 (Lester et al. 1985). W43 appears very bright and diffuse at $24 \mu \mathrm{m}$, preventing us from identifying a possible counterpart to AX J184738-0156 at this wavelength.

\subsubsection{Targeted Infrared Observations}

AX J184738-0156 is coincident with 2MASS J184736660156334, which Blum et al. (1999) resolved into a dense stellar cluster embedded within W43. We re-observed this cluster in the $J, H$, and $K_{s}$ bands using the Persson's Auxiliary Nasmyth Infrared Camera (PANIC; Martini et al. 2004; Osip et al. 2008) on the Baade, 6.5m, Magellan telescope, located at Las Campanas Observatory, on 2007 June 25. As shown in Figure 1(b), the X-ray source AX J184738-0156 is coincident with the brightest member of the star cluster. Blum et al. (1999) obtained a $K$-band spectrum of this star at a spectral resolution of $R \approx 800$, classifying it as a WR star of subtype WN7; this star is now designated WR 121a (van der Hucht 2001).

We obtained $K$-band $2.03-2.30 \mu \mathrm{m}$ spectra of the counterparts to AX J163252-4746, AX J144701-5919, and AX J144547-5931 on 2008 June 15 and 16 and 2009 July 20, respectively, at the $4.1 \mathrm{~m}$ Southern Observatory for Astrophysical Research (SOAR) Telescope, located on Cerro Pachon in Chile. The Ohio State Infrared Imager/Spectrometer (OSIRIS; Depoy et al. 1993) was used to observe AX J163252-4746 and AX J144701-5919 in the high-resolution longslit mode, which provides a spectral resolution of $R \approx 3000$ in the $K$ band. Stellar spectra were acquired in a slit-scan sequence of 5 positions separated by $5^{\prime \prime}$ each. We used the same instrument to observe AX J144547-5931 in the cross-dispersed mode at a resolution of $R \approx 1200$, using a $10^{\prime \prime}$ slit scan with 4 repetitions. Standard reduction procedures, including telluric correction, were applied to the data.

The $K$-band spectrum of AX J163252-4746, shown in Figure 3(a), exhibits an extremely strong emission line of $\mathrm{He}_{\mathrm{I}} \lambda 2.058 \mu \mathrm{m}$, as well as strong emission features from the $\lambda 2.112-2.115 \mu \mathrm{m}$ complex, composed of He I, N III, C III, and $\mathrm{O}$ III, and $\mathrm{Br} \gamma / \mathrm{He} \mathrm{I} \lambda 2.166 \mu \mathrm{m}$. The lower panel of Figure 3(a) is a magnified view of this star's spectrum showing the presence of weaker emission features from $\mathrm{N}_{\text {III }} \lambda 2.247 \mu \mathrm{m}$, He II $\lambda 2.189 \mu \mathrm{m}$, and possibly $\mathrm{N} \mathrm{v}$ at $\lambda 2.100 \mu \mathrm{m}$.

The $K$-band OSIRIS spectra of AX J144701-5919 and AX J144547-5931 are shown in Figure 3(b). While both AX J144701-5919 and AX J144547-593 show clear emission lines, the spectrum of the latter is poorer, as high clouds and vibrations due to strong wind led to a high background and significant flux variation between the exposures. The $K$-band spectrum of AX J184738-0156 can be found in Figure 5 of Blum et al. (1999). In all three cases the $\lambda 2.112-2.115$ complex $\mu \mathrm{m}$ and $\mathrm{Br} \gamma /$ He I $\lambda 2.166 \mu \mathrm{m}$ are seen in emission. AX J144701-5919 and AX J184738-0156 also have a strong He II emission line at $\lambda 2.189 \mu \mathrm{m}$. While AX J184738-0156 shows He I $\lambda 2.058 \mu \mathrm{m}$ in emission, for AX J144701-5919 this line has a P Cygni profile, with a weak emission, but strong absorption component. AX J144547-0593 displays the C IV $\lambda 2.070-2.084 \mu \mathrm{m}$ emission line complex. The atomic transitions and center wavelengths of these emission lines can be found in Morris et al. (1996).

\subsubsection{Targeted Optical Observations}

A low signal-to-noise optical spectrum of AX J1445475931 was obtained with the Low Dispersion Survey Spectrograph (LDSS-3; Osip et al. 2008) on the 6.5m Clay Magellan telescope, on 2008 June 23. This source was observed using the VPH_ALL (400 lines/mm) grism, with a center slit, resulting in a wavelength range of 3000-11000 $\mathrm{A}$. This spectrum shows $\mathrm{H} \alpha$ in emission, with C III $\lambda 5696$ and $\mathrm{C}_{\text {III }} \lambda 6721,6727,6731 \AA$ emission lines. Figure 3(c) displays the part of the LDSS-3 spectrum with these emission lines.

\subsubsection{Radio Observations}

AX J163252-4746, AX J144701-5919, and AX J1445475931 are coincident with radio emission in the first and second 

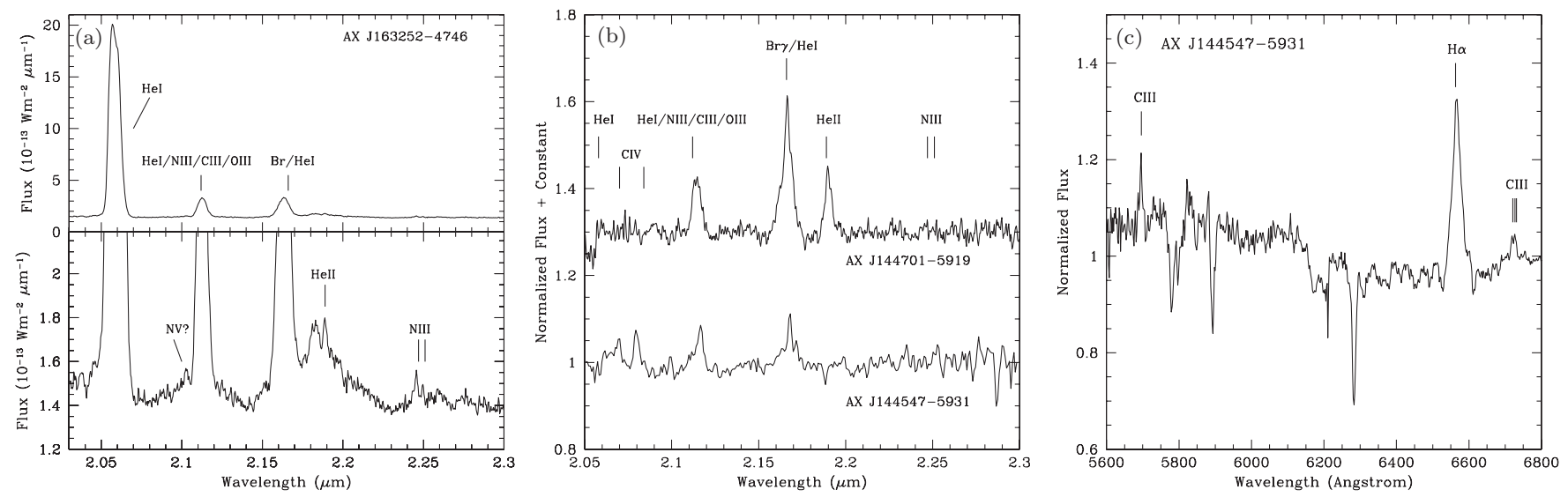

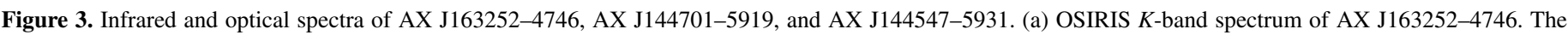

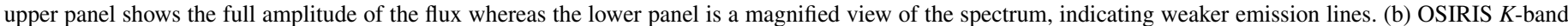
spectra of AX J144701-5919 and AX J144547-5932. (c) LDSS3 optical spectrum of AX J144547-5931.

epoch Molonglo Galactic Plane Surveys (MGPS1 and MGPS2, respectively; Green et al. 1999; Murphy et al. 2007). The MGPS data sets are made up of mosaic images, taken at $843 \mathrm{MHz}$ with the Molonglo Observatory Synthesis Telescope (MOST).

AX J144701-5919 appears to be associated with an unresolved radio source. Molonglo observed this source in 1994 and 1998 , with a measured flux density of $15 \pm 5$ and $10 \pm 5 \mathrm{mJy}$, respectively. Within these large errors there is no evidence of flux variability between these epochs. AX J163252-4746 and AX J144547-5931 fall within regions of extended radio emission. AX J184738-0156 is coincident with a bright knot within diffuse radio emission from the surrounding $\mathrm{H}_{\mathrm{II}}$ region W43, as seen at $1.4 \mathrm{GHz}$ in the Multi-Array Galactic Plane Imaging Survey (MAGPIS; Helfand et al. 2006).

We used the Australia Telescope Compact Array (ATCA) to follow-up the counterpart to AX J144701-5919 and resolve out the diffuse radio emission surrounding AX J163252-4746, observing each source for one hour at each of 1.4, 2.4, 4.8 and $8.6 \mathrm{GHz}$ with a $6 \mathrm{~km}$ baseline configuration on 2008 January 21 (AX J144701-5818) and 2008 April 11 (AX J163252-4746). ${ }^{20}$ The radio counterpart to AX J144701-5919 was detected as an unresolved point source in all 4 ATCA frequency bands, with a flux that decreased with frequency according to $S_{v}^{\text {total }} \approx$ $\nu^{\alpha}$ with $\alpha=-0.54 \pm 0.09$. AX J163252-4746 was only detected at 4.8 and $8.6 \mathrm{GHz}$ resulting in a spectral index of $\alpha=+0.19 \pm 0.02$. The observed ATCA radio fluxes and upper limits for AX J163252-4746 and AX J144701-5919 are listed in Table 3. Further high-resolution radio follow-up is required to determine whether AX J184738-0156 and AX J144547-5931 have compact radio counterparts.

\section{DISCUSSION}

\subsection{Classification of Stellar Counterparts}

The infrared colors of AX J163252-4746, AX J184738-0156, AX J144701-5919, and AX J144547-5931 are reasonably consistent with the Hadfield et al. (2007) and Mauerhan et al. (2009) selection criteria for WR stars, i.e., GLIMPSE colors [3.6] $-[8.0]>0.5$ and [3.6] $-[4.5]>0.1$ and a

${ }^{20}$ During the observation of AX J163252-4746 the antenna providing the $6 \mathrm{~km}$ baseline was not operational at 1.4 and $2.4 \mathrm{GHz}$. This resulted in $3 \mathrm{~km}$ being the longest baseline during the observations of this source at these frequencies.
2MASS+GLIMPSE color of $K-[0.8]>0.7$. This color selection criteria takes advantage of the free-free excess emission that is generated within the strong, dense, ionized winds of WR stars. The colors of our four target, thus, indicate the presence of such winds. The PAH ring surrounding AX J163252-4746 (Figure 1(a)) is also consistent with a strong wind being generated by the central source.

The $K$-band spectrum of AX J184738-0156 obtained by Blum et al. (1999) and our OSIRIS $K$-band spectra of AX J163252-4746, AX J144701-5919, and AX J144547-5931 all show strong emission lines from the $\lambda 2.112-2.115 \mu \mathrm{m}$ complex and $\mathrm{Br} \gamma / \mathrm{He}$ I $\lambda 2.166 \mu \mathrm{m}$. The presence of such emission lines are indicative of these stars being massive $\left(>20 M_{\odot}\right)$, from either the late-type WR stars in the nitrogen sequence (WN) that are hydrogen-rich or Of stars, particularly of the $\mathrm{OIf}^{+}$variety (Martins et al. 2008). WR is the name traditionally given to hydrogen-poor, emission line stars, however, there is a subset of the nitrogen sequence WR stars that have hydrogen in their spectra. Smith \& Conti (2008) proposed a new designation for this subset, WNH, as they are different from classical WR stars in that they are still undergoing hydrogen-core burning and are therefore at an earlier stage in their evolution. WNH stars are some of the most massive in our Galaxy, with a mass distribution that peaks around $\sim 50 M_{\odot}$, but which can reach as high as $120 M_{\odot}$ (Smith \& Conti 2008), making them very short-lived and rare. They can have luminosities as high as $2 \times 10^{6} L_{\odot}$ and large mass-loss rates of $\dot{M}>10^{-5} M_{\odot} \mathrm{yr}^{-1}$ due to fast extended winds (Martins et al. 2008). OIf $^{+}$stars are also extremely massive with very similar properties (Martins et al. 2008). Both WNH and $\mathrm{OIf}^{+}$stars are known X-ray emitters (e.g., Mauerhan et al. 2010).

WNH and $\mathrm{OIf}^{+}$stars also produce other spectral line features allowing us to distinguish between the different subtypes. The $K$-band spectrum of AX J163252-4746 (Figure 3(a)) is remarkable, in that it exhibits an ultra-strong Не I $\lambda 2.058 \mu \mathrm{m}$ emission line. The total EW is $\approx 1040 \AA$, with an FWHM of $\approx 74 \AA\left(1080 \mathrm{~km} \mathrm{~s}^{-1}\right)$. The spectrum also exhibits strong emission features from the $\lambda 2.112-2.115 \mu \mathrm{m}$ complex $(\mathrm{EW} \approx 80 \AA$ ) and $\mathrm{Br} \gamma / \mathrm{He}$ I $\lambda 2.166 \mu \mathrm{m}(\mathrm{EW} \approx 90 \AA)$. The relative strengths of the emission lines, especially the very strong He I emission, are similar to those of the Ofpe/WN9 stars WR 122 and WR 85a (Morris et al. 1996; Figer et al. 1997), although the individual line strengths are significantly stronger for 
AX J163252-4746 than for these other sources. Ofpe/WN9 stars, also known as "slash stars," have been described as both WNHs or luminous blue variables (Smith \& Conti 2008). We assign AX J163252-4746 the spectral type Ofpe/WN9.

The zoomed view of AX J163252-4746, seen in the lower panel of Figure 3(a), show fainter emission features that could indicate the presence of a massive companion. There is a spectral hint of broad He I $\lambda 2.112 \mu \mathrm{m}$ emission under the narrower line of the brighter star and a broad spectral feature from He II $\lambda 2.189 \mu \mathrm{m}$. Though He II $\lambda 2.189 \mu \mathrm{m}$ is a known emission feature of Ofpe/WN9 stars, the broadness of this line and the He I feature are both reminiscent of an early-type WN star (WNE) of the WN4-6 variety (Figer et al. 1997). If these features are real, their weakness may be due to the fact that WNE stars are up to two magnitudes fainter than Ofpe/WN9 stars, intrinsically (Crowther et al. 2006). The questionable weak feature near $\lambda 2.10 \mu \mathrm{m}$, speculated to be $\mathrm{N} \mathrm{v}$ emission, could also indicate the presence of an underlying WNE spectrum since this line requires the associated high temperatures of a WNE star. The weak N III $\lambda 2.247 \mu \mathrm{m}$ doublet could be from either star, though is more ordinarily seen in late-type WNs, which include the WN7-9 subtypes (Figer et al. 1997; Martins et al. 2008). Finally, as a separate speculation, we note that the shape of the peak in the He I $\lambda 2.058 \mu \mathrm{m}$ line, seen in the top panel of Figure 3(a), appears to have a double peaked profile, as if it were a blend of two velocity-shifted components having comparable line strengths. However, none of the other strong lines exhibit this profile. Given the current data this is all very speculative. We defer any definitive conclusions about the weak spectral features or the potential contribution of a companion spectrum until further observations are conducted. Higher resolution spectroscopic measurements over several epochs will be required to determine if the weak, underlying, broad features are real, and to identify this source as a spectroscopic binary.

As noted in Section 2.2.2, AX J184738-0156 is coincident with WR 121a, which was identified by Blum et al. (1999) as a star of subtype WN7. The $K$-band spectrum of this star, seen in Figure 5 of Blum et al. (1999), is similar to WN7-8h stars, which are WNH subtypes (e.g., see Martins et al. 2008; Mauerhan et al. 2010). X-ray emission has not been connected with WR 121a prior to this paper. However, there is evidence to suggest that WR 121a and its surrounding star-formation region, W43, may be associated with the extended (intrinsic rms size of $0.32 \pm 0.02)$ very high energy $(E>100 \mathrm{GeV})$ $\gamma$-ray source HESS J1848-018 (Chaves et al. 2008) and its possible counterpart, OFGL J1848.6-0138, detected with the Large Area Telescope on board the Fermi Gamma-ray Space Telescope (Tam et al. 2010). W43 is also host to the bright HII region G30.8-0.2 and a giant molecular cloud, which partially overlaps with HESS J1848-018, their centroids separated by $\sim 0.3$. WR 121a lies within the region of this HESS source's extended emission, $\sim 0.2$ from its centroid (Chaves et al. 2008). A similar offset exists between HESS J1023-575 and the young open cluster, Westerlund 2, host to WR 20a (Aharonian et al. 2007) yet the extended nature of this HESS source, and therefore HESS J1848-018, argues against a single star origin for these VHE $\gamma$-ray sources (Aharonian et al. 2007; Chaves et al. 2008).

For AX J144701-5919 the $\mathrm{Br} \gamma / \mathrm{He}_{\mathrm{I}}$ emission line in the $K$-band spectrum (Figure $3(\mathrm{~b})$ ) is stronger than the $\lambda 2.112-2.115 \mu \mathrm{m}$ emission line complex indicating a spectral type later than WN6. As the He II $\lambda 2.189 \mu \mathrm{m}$ feature is also seen in emission we expect a spectral type earlier than WN8-9 as this feature would otherwise appear in absorption or would have a
P Cygni profile (e.g., see Martins et al. 2008). The ratio of EWs of the detected lines can be used to quantitatively determine the WN subtype. We measure $\operatorname{EW}(\lambda 2.189 \mu \mathrm{m}) / \mathrm{EW}(\lambda 2.112 \mu \mathrm{m})$ $=0.4$ and $\operatorname{EW}(\lambda 2.189 \mu \mathrm{m}) / \operatorname{EW}(\lambda 2.166 \mu \mathrm{m})=0.3$, which are most consistent with WN7-8 subtypes (Figer et al. 1997; Martins et al. 2008). We therefore classify the counterpart to AX J144701-5919 as a WNH star of subtype WN7-8h.

The $K$-band spectrum of AX J144547-5931 (Figure 3(b)) shows He II $\lambda 2.189 \mu \mathrm{m}$ in absorption, which is indicative of either a WN8-9 or an $\mathrm{OIf}^{+}$star (Martins et al. 2008). As the $\lambda 2.112-2.115 \mu \mathrm{m}$ complex and the $\mathrm{Br} \gamma / \mathrm{He} \mathrm{I} \lambda 2.166 \mu \mathrm{m}$ emission line are of similar strength it is more likely an $\mathrm{OIf}^{+}$ subtype (Martins et al. 2008). The LDSS-3 optical spectrum of AX J144547-5931 (Figure 3(c)) is missing the He I $\lambda 6678 \mu \mathrm{m}$ emission line seen very strongly in optical spectra of WN8-9 stars (e.g., see Corradi et al. 2010). It does have a very broad $\mathrm{H} \alpha$ line, as well as C III $\lambda 5696 \AA$ and $\mathrm{C}_{\mathrm{III}} \lambda 6721,6727$, $6731 \AA$ emission, which are known O-type star spectral lines (Walborn 2001). These spectral features are characteristic of $\mathrm{OIf}^{+}$stars identified by De Becker et al. (2006, 2009). Further spectroscopic follow-up is required to constrain the $\mathrm{OIf}^{+}$subtype of AX J144547-5931. This could be achieved by the detection of Si IV emission at $\lambda 4089-4116 \AA$, which is, historically, the defining characteristic of high-luminosity $\mathrm{OIf}^{+}$stars (Walborn 1971).

\subsection{Distances and $X$-ray Luminosities}

Stars with the spectral classification of AX J163252-4746, Ofpe/WN9, have a narrow range in absolute $K$-band magnitude $\left(M_{K}\right)$, which can be combined with an estimate of the extinction along the line of sight to determine the approximate distance to the source. This method is outlined and tabulated by Hadfield et al. (2007) and Mauerhan et al. (2009), and adopts intrinsic colors from Crowther et al. (2006). Assuming the colors and $M_{K}$ value for a WN10-11 star (aka Ofpe/WN9) from Crowther et al. (2006) we derive an approximate distance of $4.9 \mathrm{kpc}$. Using red clump stars to measure the reddening as a function of distance along the line of site (López-Corredoira et al. 2002) we can also calculate a lower limit of $\gtrsim 4.5 \mathrm{kpc}$ on the distance to AX $\mathrm{J} 163252-4746$, which is consistent with our result. We therefore adopt a distance of $\sim 4.9 \mathrm{kpc}$ to AX J163252-4746 yielding an unabsorbed X-ray luminosity of $L_{x} \approx 3.4 \times 10^{34} \mathrm{erg} \mathrm{s}^{-1}$ in the $0.3-8.0 \mathrm{keV}$ energy range.

The kinematic distance to W43, in which AX J184738-0156 is embedded, is $5.6 \mathrm{kpc}$ (Smith et al. 1978). ${ }^{21}$ This is also consistent with the red clump lower limit of $\gtrsim 3.5 \mathrm{kpc}$. For a distance of $5.6 \mathrm{kpc}$ to $\mathrm{AX}$ J184738-0156 we calculate an unabsorbed luminosity of $L_{x} \approx 3.4 \times 10^{34} \mathrm{erg} \mathrm{s}^{-1}$ in the 0.3-8.0 keV energy range.

AX J144701-5919 and AX J144547-5931 are separated on the sky by only $\sim 16^{\prime}$ and are coincident with a star formation complex. There are close surrounding $\mathrm{H}$ II regions, G316.808-0.037, G317.037+0.300 and G317.291+0.268, for which Caswell \& Haynes (1987) used radio recombination line velocities to calculate a near and a far kinematic distance to each. As Shaver et al. (1981) present radio and infrared observations indicating that G316.808-0.037 is located at the closer kinematic distance of $\sim 2.3 \mathrm{kpc}$ we assume the nearer distance for the other two regions also, so that G317.037+0.300 and G317.291+0.268 lie at $\sim 2.8$ and $\sim 2.9 \mathrm{kpc}$, respectively. 21 Revised for a modern distance of $\sim 8 \mathrm{kpc}$ to the Galactic center (Reid et al.
2009 ). 
Using red clump stars to measure the reddening as a function of distance along the line of site (López-Corredoira et al. 2002) we can calculate a lower limit of $\gtrsim 3.0 \mathrm{kpc}$ to these two $\mathrm{X}$-ray sources, which is reasonably consistent with the kinematic distances.

Stars with the spectral classification of AX J144701-5919, WN7-8h, also have a narrow range in absolute $K$-band magnitude. By averaging together the $M_{k}$ values of two WN7-8h stars in the Arches cluster (Martins et al. 2008) and using the Crowther et al. (2006) colors for WN7-9 stars to estimate the extinction along the line of sight, we further refine our distance estimate to $\sim 2.6 \mathrm{kpc}$ for AX J144701-5919, resulting in an unabsorbed luminosity of $L_{x} \approx 3.2 \times 10^{33} \mathrm{erg} \mathrm{s}^{-1}$ in the $0.3-8.0 \mathrm{keV}$ energy range. This distance is fairly consistent with the red-clump value and estimates via comparison with neighboring $\mathrm{H}$ II regions.

As we do not have a strong enough constraint on the $\mathrm{OIf}^{+}$luminosity class we cannot apply the same method to AX J144547-5931. We therefore derive a range of possible unabsorbed X-ray luminosities based on both the kinematic and red clump distances, $2.3-3.0 \mathrm{kpc}$. In this case $L_{x} \approx$ $1.9-3.2 \times 10^{33} \mathrm{erg} \mathrm{s}^{-1}$ in the $0.3-8.0 \mathrm{keV}$ energy range.

In each case the unabsorbed X-ray luminosity is derived from the absorbed Raymond-Smith spectral fits to the X-ray spectra. These luminosities can be found in Table 2 along with the distances used for the calculations. All these distances have been revised for a modern distance of $8 \mathrm{kpc}$ to the Galactic center (Reid et al. 2009).

\subsection{AX J163252-4746 and AX J184738-0156}

AX J163252-4746 and AX J184738-0156 are the two most luminous sources out of the four investigated in this paper. We discuss these sources together as they are both associated with WNH stars and have similar X-ray luminosities and characteristics as well as comparable observational information. There are three possibilities as to how these WNH stars can produce the X-ray emission we observe. These include X-rays that are intrinsic to the massive star, $\mathrm{X}$-rays produced through gravitation accretion in an HMXB or X-rays from shock-heated gas in a CWB. We now discuss each possibility in detail.

Many WN stars have been shown to have thermal $\mathrm{X}$-ray emission created through instability-driven wind shocks with typical temperatures around $k T \approx 0.6$ $\mathrm{keV}$ (Oskinova 2005). However, the XMM spectra of AX J163252-4746 and AX J184738-0156 (Figure 2) show that few X-ray photons were detected with energies $<1 \mathrm{keV}$, indicating high levels of absorption preventing the detection of such a stellar wind component. Raymond-Smith fits to the same spectra result in temperatures of $k T=3.2$ and $3.1 \mathrm{keV}$ for AX J163252-4746 and AX J184738-0156, respectively (see Table 2), so the majority of the observed X-ray emission from these two sources likely arises from another mechanism capable of generating a much hotter plasma.

In an isolated massive star thermal X-rays can also be generated through the magnetically channeled wind-shock mechanism. Gagné et al. (2005) have successfully demonstrated that this model can explain the high temperature $(k T \sim 3 \mathrm{keV})$ $\mathrm{X}$-ray plasma and X-ray luminosity $\left(L_{x} \approx 10^{33} \mathrm{erg} \mathrm{s}^{-1}\right.$; $0.5-10 \mathrm{keV}$ ) associated with the $05.5 \mathrm{~V}$ star $\theta^{1}$ Orionis C. However, there are problems applying this same model to WR stars as they have far stronger wind momenta than $\mathrm{O}$ stars. Mauerhan et al. (2010) showed that the WR stars and O supergiants in their sample, which have similar X-ray properties and luminosities to the four sources discussed in this paper, would require $\approx 5 \mathrm{kG}$ magnetic fields to confine the winds. Thus far no such fields have been detected around WR stars. We thus regard this scenario as very unlikely.

The generation of non-thermal X-rays in a single massive star has also been considered (e.g., Chen \& White 1991), however, recent studies of $\mathrm{O}$ stars have shown that in most cases, at energies $<10 \mathrm{keV}$, any non-thermal $\mathrm{X}$-rays are likely to make an insignificant contribution to the overall stellar X-ray spectrum (Pittard \& Parkin 2010). We assume that this is also the case for WNH stars as they are only slightly more evolved than massive O stars (Smith \& Conti 2008).

Given the above arguments it is unlikely that the majority of X-ray emission from either AX J163252-4746 or AX J184738-0156 is generated through the instability-driven or magnetically channeled wind-shock mechanisms, or through inverse Compton scattering. The X-rays are therefore not intrinsic to the WNH counterparts, so further scenarios need to be considered.

The radio spectrum of a single WR star with an optically thick stellar wind is thermal and has a spectral index of $\alpha \approx+0.7 \pm 0.1$ (Dougherty \& Williams 2000). If the wind is optically thin and has a radio spectral index $<+0.6$ this implies that there is another component to the emission. The ATCA observation of AX J163252-4746 shows its radio spectrum to be approximately flat $(\alpha \approx+0.2)$. This could indicate that there is a significant non-thermal component to the spectrum where this and the thermal component of the source are inferred to have similar luminosities. Such spectra are referred to as "composite" (Dougherty \& Williams 2000). Likely origins of non-thermal radio emission associated with massive stars are either synchrotron emission from ejected material (Fender 2001, and references therein) or from short-lived jets (e.g., Pestalozzi et al. 2009) in HMXBs or relativistic electrons accelerated in the wind collision region (WCR) of a CWB (Eichler \& Usov 1993; Dougherty et al. 2003; Pittard et al. 2006). A flat spectral index may also occur if the optically thin thermal radio emission from the WCR of a CWB is comparable to that of the thermal emission from the unshocked winds in the system (Pittard et al. 2006). The resulting spectrum would resemble that of a composite spectrum.

Of the two main classes of HMXBs, AX J163252-4746 and AX J184738-0156 are more likely to be a form of SGXB given their counterpart classification as WNH stars with infrared excess implying strong stellar winds. Most SGXBs are persistent X-ray sources with X-ray luminosities around $L_{x} \sim 10^{35}-10^{36} \mathrm{erg} \mathrm{s}^{-1}$ (Sguera et al. 2006). Currently we only know of four HMXBs with WR companions, three of which are SGXB BH candidates and only one, Cygnus X-3, is found in the Milky Way (Szostek et al. 2008). The other, OAO 1657-415, which is also the AGPS source AX J170047-4139 (Sugizaki et al. 2001), is an X-ray pulsar with an Ofpe/WN9 companion (Mason et al. 2009). This source has an X-ray luminosity of $L_{x} \sim 10^{36}-10^{37} \mathrm{erg} \mathrm{s}^{-1}$ and appears to be transitioning from a wind-fed SGXB into a BeX disk-fed system (Mason et al. 2009). "Classical" SGXBs and systems such as OAO 1657-415 are therefore too luminous to explain the X-ray emission seen in AX J163252-4746 and AX J184738-0156.

However, the unabsorbed X-ray luminosities of AX J1632524746 and AX J184738-0156 are far more consistent with the quiescent luminosities of a subclass of SGXBs that have recently been discovered with the Integral satellite, called supergiant fast X-ray transients (SFXTs; Sguera et al. 2006). These systems 
are neutron star HMXBs that produce extremely bright, rapid X-ray bursts with luminosities reaching $L_{x} \sim 10^{36} \mathrm{erg} \mathrm{s}^{-1}$ (Chaty 2010) and have quiescent luminosities at or below $L_{x} \sim 10^{32}-10^{33} \mathrm{erg} \mathrm{s}^{-1}$ (Sguera et al. 2006). Currently very few sources with X-ray bursts have been confirmed as SFXTs, most of which contain X-ray pulsars (Negueruela et al. 2006; Sguera et al. 2006). The X-ray luminosities of AX J163252-4746 and AX J184738-0156, listed in Table 2, are at least an order of magnitude brighter than the quiescent luminosities of SFXTs and the X-ray flux observed for each source during the ASCA, $X M M$, and Chandra observations shows no evidence for longterm variability. A thorough investigation of the $X M M$ and Chandra observations also show no evidence of short-term variability, flaring or pulsation. SFXTs also have hard, nonthermal X-ray spectra that are well described by a power law with a photon index of $\Gamma \sim 1$ (Lutovinov et al. 2005). Powerlaw fits to the XMM spectra of AX J163252-4746 and AX J184738-0156 are much steeper than this with photon indices of $\Gamma \sim 3$.

As the XMM spectra of AX J163252-4746 and AX J184738-0156 both have a strong Fe XXV emission line at $\sim 6.7 \mathrm{keV}$, indicating the presence of very high temperature plasma, these spectra are better described by a thin thermal plasma model, not typically seen in HMXBs. While low iron ionization state emission lines (Fe I-Fe XII) have been seen in many HMXB X-ray spectra (e.g., Tomsick et al. 2009), created by cool accretion disks irradiated by a high-energy source (Caballero-García et al. 2009), it is hard to explain the presence of the $\sim 6.7 \mathrm{keV} \mathrm{Fe} \mathrm{XXV}$ in the X-ray spectra of these two sources. This line can only be created in an environment capable of producing a very highly ionized plasma (Reig 1999). Given this fact, combined with the other X-ray properties of AX J163252-4746 and AX J184738-0156, an HMXB interpretation does not seem viable.

We now consider a CWB interpretation for these two sources. The X-ray luminosities from WNH CWBs are usually between $L_{x} \sim 10^{32}$ and $10^{34} \mathrm{erg} \mathrm{s}^{-1}$ (Mauerhan et al. 2010). Our estimates of the unabsorbed luminosities of AX J163252-4746 and AX J184738-0156 are consistent with this range. The very hot thermal plasma $(k T>3 \mathrm{keV})$ seen in the Raymond-Smith fits to the XMM spectra of AX J163252-4746 and AX J184738-0156 indicates binarity, where the hard X-ray emission could be generated by colliding supersonic winds in a CWB (Prilutskii \& Usov 1976; Cherepashchuk 1976; Usov 1992).

The interface between the winds of two massive stars is capable of generating the hot thermal plasma necessary to produce the $6.7 \mathrm{keV} \mathrm{Fe} \mathrm{XXV} \mathrm{emission} \mathrm{line} \mathrm{seen} \mathrm{in} \mathrm{the} \mathrm{XMM}$ spectra of AX J163252-4746 and AX J184738-0156. Raassen et al. (2003) argue that for WR 25, another WNH star classified

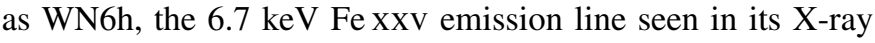
spectrum can only be created in a wide binary, made up of this star and a massive WR or O star companion, with their winds thus colliding at speeds close to their terminal velocities of over $1000 \mathrm{kms}^{-1}$ (van der Hucht 2001). It should be noted here that this might not be true for all WR CWBs (see Zhekov \& Park 2010). As both AX J163252-4746 and AX J184738-0156 show a lack of variability and periodicity in X-rays, have thermal $\mathrm{X}$-ray plasma temperatures and luminosities similar to known WNH CWBs, and also display the $6.7 \mathrm{keV} \mathrm{Fe} \mathrm{XxV} \mathrm{emission} \mathrm{line}$ in their X-ray spectra, we classify these two sources as CWBs.

The same relationship that we see between the X-ray and bolometric luminosities of single $\mathrm{O}$ stars, attributed to instability-driven wind shocks, has been shown to hold for $\mathrm{WN}+\mathrm{O}$ and $\mathrm{O}+\mathrm{O}$ binaries, where $\log \left(L_{x} / L_{\text {bol }}\right) \approx-7$ (Oskinova 2005; Mauerhan et al. 2010). This suggests that a major fraction of the X-rays from CWBs are soft $(<2.5 \mathrm{keV})$, emitted by the individual stellar winds rather than in the WCR (Oskinova 2005; Antokhin et al. 2008). That being said, there is no relationship known to exist for putatively single WN stars (Oskinova 2005; Mauerhan et al. 2010) so it is still perplexing as to why there are not more luminous massive binaries where large amounts of hard X-rays are generated in the WCR (Antokhin et al. 2008). There are therefore few cases where CWBs are overluminous, with $\log \left(L_{x} / L_{\text {bol }}\right)>-7$. We calculated a range of $\log \left(L_{x} / L_{\text {bol }}\right)$ for $\mathrm{AX} \mathrm{J} 163252-4746$ and $\mathrm{AX}$ J184738-0156 by averaging the $L_{\text {bol }}$ values of the Ofpe/WN9 stars listed in Oskinova (2005) and Mauerhan et al. (2010) for AX J163252-4746 and the WN7-8h stars in Mauerhan et al. (2010) for AX J184738-0156. For both sources we find $\log \left(L_{x} / L_{\text {bol }}\right) \gtrsim-5.4$, as listed in Table 2 . There are very few examples of WN CWBs where $\log \left(L_{x} / L_{\text {bol }}\right)>-6$ and these include CXOGC J174550.2-284911, a WN8-9h CWB with $\log \left(L_{x} / L_{\text {bol }}\right) \sim-5.8$ (Mauerhan et al. 2010), and WR 25 (WN6ha+04f) with $\log \left(L_{x} / L_{\text {bol }}\right) \sim-5.6$ (Raassen et al. 2003). AX J163252-4746 and AX J184738-0156 therefore appear to be extremely X-ray luminous CWBs.

\subsection{AX J144701-5919 and AX J144547-5931}

AX J144701-5919 and AX J144547-5931 are much fainter and somewhat softer than AX J163252-4746 and AX J184738-0156 but all four sources have similar massive counterparts. We now address the isolated massive star, HMXB and CWB scenarios for AX J144701-5919 and AX J144547-5931.

Due to the low number of counts in their Chandra observations we cannot obtain a reliable spectral fit or identify any X-ray emission lines in the X-ray spectra of AX J144701-5919 and AX J144547-5931. Instead we explore the hardness of their Xray spectra by seeing how the $50 \%$ and $75 \%$ photon energy $\left(E_{50}\right.$ and $E_{75}$, respectively), the energy below which $50 \%$ and $75 \%$ of the photons energies are found, of these two sources compare to the same values for AX J163252-4746 and AX J184738-0156. The $E_{50}$ value indicates whether a given source is hard $\left(E_{50}>2\right.$ $\mathrm{keV})$ or soft $\left(E_{50}<2 \mathrm{keV}\right)$ and $E_{75} \mathrm{keV}$ grades the level of hardness within the hard category. In all four cases $E_{50}$ is between 2 and $3 \mathrm{keV}$. The $E_{75}$ value of AX J163252-4746, AX J184738-0156, AX J144701-5919, and AX J144547-5931 are $\sim 4.4, \sim 4.5, \sim 2.9$ and $\sim 2.9 \mathrm{keV}$, respectively. Through this crude comparison it appears that the overall spectra of AX J144701-5919 and AX J144547-5931 may be reasonably soft, consistent with the X-rays primarily being generated through instability-driven wind shocks, intrinsic to the individual stars.

The X-ray luminosities of AX J144701-5919 and AX J144547-5931, listed in Table 2, are slightly brighter than many putatively single WN and O stars (e.g., Oskinova 2005; Antokhin et al. 2008) but are consistent with the X-ray luminosities of CWBs and quiescent SFXTs. However, using the same method as described in Section 3.3, we calculated a range of $\log \left(L_{x} / L_{\text {bol }}\right)$ for both sources, listed in Table 2 . In each case $\log \left(L_{x} / L_{\text {bol }}\right)>-6.5$ making these sources overluminous compared to the $\log \left(L_{x} / L_{\text {bol }}\right) \approx-7$ relationship seen to be exhibited by most single $\mathrm{O}$ stars and $\mathrm{WN}$ and $\mathrm{O}$ star massive binaries. This does not narrow down our identification as many examples of overluminous $\left(\log \left(L_{x} / L_{\text {bol }}\right)>-7\right)$ putatively single $\mathrm{WN}$ and O stars, as well as CWBs, with $\log \left(L_{x} / L_{\text {bol }}\right) \approx-6.5$, have been 
detected (e.g., Oskinova 2005; Sana et al. 2006; Antokhin et al. 2008; Mauerhan et al. 2010).

There is no evidence to suggest long-term variability between the original ASCA and recent Chandra observations of AX J144701-5919 and AX J144547-5931. This, combined with the lack of short-term variability or periodicity down to $6.4 \mathrm{~s}$ seen in the Chandra observations, is more supportive of a single star or CWB scenario rather than an SFXT scenario. However, longer X-ray observations are required to confirm this interpretation.

The radio spectral index of AX J144701-5919 is negative $(\alpha \approx-0.5)$ and therefore dominated by non-thermal emission. This spectral index predicts a flux of $\sim 38 \mathrm{mJy}$ at $843 \mathrm{MHz}$, which is much higher than the fluxes measured by MOST at this frequency $(\sim 10-15 \mathrm{mJy})$. This discrepancy could be due to time variability, or the radio spectrum may turn over at lower frequencies in a similar fashion to WR 147 (Skinner et al. 1999). Further radio observations are required to distinguish between these two scenarios. Even though non-thermal radio emission supports a binary interpretation for the WN7-8h counterpart to AX J144701-5919, it does not allow us to discriminate between the possible origins of the X-ray emission.

In summary, the Chandra observations of AX J144701-5919 and AX J144547-5931 show these sources to have similar Xray luminosities to those seen in putatively single massive stars, CWBs and quiescent SFXTs. As $\log \left(L_{x} / L_{\text {bol }}\right)>-6.5$ for both these sources, this supports both a binary and X-ray luminous isolated massive star scenario, the later of which is also supported by the fact that few hard X-ray photons $(k T>3 \mathrm{keV})$ were detected. Longer X-ray observations to obtain statistically significant spectra are required to determine the mechanisms behind the X-ray generation from these two massive stars.

\section{CONCLUSIONS}

We have characterized the multi-wavelength properties of four previously unidentified AGPS sources, AX J163252-4746, AX J184738-0156, AX J144701-5919, and AX J144547-5931, identifying them as X-ray-emitting massive stars. Our data are most consistent with CWB classifications for AX J163252-4746 and AX J184738-0156. AX J144547-5931 and AX J144701-5919 require longer X-ray observations to distinguish between a CWB, an HMXB, or an extremely X-ray luminous isolated massive star.

In the full ChIcAGO survey we have so far observed 95 AGPS sources with Chandra, $10 \%$ of which emit hard $\mathrm{X}$-rays (median energies $>2 \mathrm{keV}$ ) and have colors and infrared excess similar to the four objects discussed in this paper. One AGPS source with these characteristics, AX J183116-1008, has indeed recently been identified as a WN8 binary object, likely a CWB, with an unabsorbed X-ray luminosity of $L_{x} \sim$ $1.3 \times 10^{34} \mathrm{erg} \mathrm{s}^{-1}$ (Motch et al. 2010). It is possible that the other AGPS sources with similar X-ray and infrared properties to the four discussed in this paper could be more X-ray-emitting CWBs or X-ray binary objects.

The counterparts to AX J163252-4746, AX J184738-0156, AX J144701-5919, and AX J144547-5931 as well as AX J183116-1008, are all extremely massive stars, making them short-lived and therefore rare, absorbed and only found in the Galactic plane. This makes the AGPS and our follow-up ChIcAGO project, an efficient way of locating and identifying massive stars in our Galaxy.
Special thanks goes to Michael Muno for his encouragement, expertise, and participation in this project. We also thank Sean Farrell, Stan Owocki, and Nathan Smith for their advice and help with this research and the referee for his or her constructive response and suggestions. G.E.A acknowledges the support of an Australian Postgraduate Award. B.M.G. acknowledges the support of a Federation Fellowship from the Australian Research Council through grant FF0561298. D.L.K. was supported by NASA through Hubble Fellowship grant 51230.01-A awarded by the STScI, which is operated by AURA, for NASA, under contract NAS 5-26555. P.O.S. acknowledges partial support from NASA Contract NAS803060. D.T.H.S. acknowledges an STFC Advanced Fellowship. J.J.D was supported by NASA contract NAS8-39073 to the Chandra X-ray Center (CXC). Basic research in radio astronomy at the NRL is supported by 6.1 Base funding. Support for this work was also provided by NASA through Chandra Award Number GO9-0155X issued by the CXC, which is operated by the Smithsonian Astrophysical Observatory for and on behalf of NASA. This research makes use of data obtained with the Chandra X-ray Observatory, and software provided by the CXC in the application packages CIAO and Sherpa. OSIRIS is a collaborative project between Ohio State University and CTIO. Observing time on the $6.5 \mathrm{~m}$ Clay and Baade Magellan Telescopes, located at Las Campanas Observatory, was allocated through the Harvard-Smithsonian Center for Astrophysics. The ATCA, part of the Australia Telescope, is funded by the Commonwealth of Australia for operation as a National Facility managed by CSIRO. This publication makes use of data products from the second catalogue of serendipitous $\mathrm{X}$-ray sources (2XMMi) from the European Space Agency's (ESA) XMM-Newton observatory. These data were accessed through the Leicester Database and Archive Service at Leicester University, UK. 2MASS is a joint project of the University of Massachusetts and the IPAC/Caltech, funded by the NASA and NFS. GLIMPSE survey data are part of the Spitzer Legacy Program. The Spitzer Space Telescope is operated by the JPL/ Caltech under a contract with NASA. This research has made use of NASA's Astrophysics Data System.

Facilities: ASCA, ATCA, CXO (ACIS, HRC), Magellan:Baade (PANIC), Magellan:Clay (LDSS-3), Molonglo Observatory, SOAR (OSIRIS), XMM (EPIC)

\section{REFERENCES}

Aharonian, F., et al. 2007, A\&A, 467, 1075

Anders, E., \& Grevesse, N. 1989, Geochim. Cosmochim. Acta, 53, 197

Antokhin, I. I., Rauw, G., Vreux, J., van der Hucht, K. A., \& Brown, J. C. 2008, A\&A, 477, 593

Babel, J., \& Montmerle, T. 1997a, ApJ, 485, L29

Babel, J., \& Montmerle, T. 1997b, A\&A, 323, 121

Benaglia, P., \& Romero, G. E. 2003, A\&A, 399, 1121

Benjamin, R. A., et al. 2003, PASP, 115, 953

Blum, R. D., Damineli, A., \& Conti, P. S. 1999, AJ, 117, 1392

Caballero-García, M. D., Miller, J. M., Trigo, M. D., Kuulkers, E., Fabian, A.

C., Mas-Hesse, J. M., Steeghs, D., \& van der Klis, M. 2009, ApJ, 692, 1339 Carey, S. J., et al. 2009, PASP, 121, 76

Caswell, J. L., \& Haynes, R. F. 1987, A\&A, 171, 261

Chaty, S. 2010, in ASP Conf. Ser. 422, High Energy Phenomena in Massive Stars, ed. J. Martí, P. L. Luque-Escamilla, \& J. A. Combi (San Francisco, CA: ASP), 243

Chaves, R. C. G., Renaud, M., Lemoine-Goumard, M., \& Goret, P. 2008, in AIP Conf. Ser. 1085, High Energy Gamma-Ray Astronomy, ed. F. A. Aharonian, W. Hofmann, \& F. Rieger (Melville, NY: AIP), 372

Chen, W., \& White, R. L. 1991, ApJ, 366, 512

Cherepashchuk, A. M. 1976, Sov. Astron. Lett., 2, 138

Churchwell, E., et al. 2006, ApJ, 649, 759

Churchwell, E., et al. 2007, ApJ, 670, 428 
Corradi, R. L. M., et al. 2010, A\&A, 509, A41

Crowther, P. A. 2008, in IAU Symp. 250, ed. F. Bresolin, P. A. Crowther, \& J. Puls (Cambridge: Cambridge Univ. Press), 47

Crowther, P. A., Hadfield, L. J., Clark, J. S., Negueruela, I., \& Vacca, W. D. 2006, MNRAS, 372, 1407

Davis, J. E. 2001, ApJ, 562, 575

De Becker, M. 2007, A\&AR, 14, 171

De Becker, M., Rauw, G., \& Linder, N. 2009, ApJ, 704, 964

De Becker, M., Rauw, G., Manfroid, J., \& Eenens, P. 2006, A\&A, 456, 1121

Depoy, D. L., Atwood, B., Byard, P. L., Frogel, J., \& O’Brien, T. P. 1993, Proc. SPIE, 1946, 667

Dorman, B., \& Arnaud, K. A. 2001, in ASP Conf. Ser. 238, Astronomical Data Analysis Software and Systems X, ed. F. R. Harnden, F. A. Primini, Jr., \& H. E. Payne (San Francisco, CA: ASP), 415

Dougherty, S. M., Pittard, J. M., Kasian, L., Coker, R. F., Williams, P. M., \& Lloyd, H. M. 2003, A\&A, 409, 217

Dougherty, S. M., \& Williams, P. M. 2000, MNRAS, 319, 1005

Eichler, D., \& Usov, V. 1993, ApJ, 402, 271

Fender, R. P. 2001, MNRAS, 322, 31

Figer, D. F., McLean, I. S., \& Najarro, F. 1997, ApJ, 486, 420

Gagné, M., Oksala, M. E., Cohen, D. H., Tonnesen, S. K., ud-Doula, A., Owocki, S. P., Townsend, R. H. D., \& MacFarlane, J. J. 2005, ApJ, 628, 986

Garmire, G. P., Bautz, M. W., Ford, P. G., Nousek, J. A., \& Ricker, G. R., Jr. 2003, Proc. SPIE, 4851, 28

Green, A. J., Cram, L. E., Large, M. I., \& Ye, T. 1999, ApJS, 122, 207

Grindlay, J. E., et al. 2005, ApJ, 635, 920

Hadfield, L. J., van Dyk, S. D., Morris, P. W., Smith, J. D., Marston, A. P., \& Peterson, D. E. 2007, MNRAS, 376, 248

Hands, A. D. P., Warwick, R. S., Watson, M. G., \& Helfand, D. J. 2004, MNRAS, 351,31

Helfand, D. J., Becker, R. H., White, R. L., Fallon, A., \& Tuttle, S. 2006, AJ, 131,2525

Lester, D. F., Dinerstein, H. L., Werner, M. W., Harvey, P. M., Evans, N. J., II, \& Brown, R. L. 1985, ApJ, 296, 565

López-Corredoira, M., Cabrera-Lavers, A., Garzón, F., \& Hammersley, P. L. 2002, A\&A, 394, 883

Lucy, L. B. 1982, ApJ, 255, 286

Lucy, L. B., \& White, R. L. 1980, ApJ, 241, 300

Lutovinov, A., Revnivtsev, M., Gilfanov, M., Shtykovskiy, P., Molkov, S., \& Sunyaev, R. 2005, A\&A, 444, 821

Martini, P., Persson, S. E., Murphy, D. C., Birk, C., Shectman, S. A., Gunnels, S. M., \& Koch, E. 2004, Proc. SPIE, 5492, 1653

Martins, F., Hillier, D. J., Paumard, T., Eisenhauer, F., Ott, T., \& Genzel, R. 2008, A\&A, 478, 219

Mason, A. B., Clark, J. S., Norton, A. J., Negueruela, I., \& Roche, P. 2009, A\&A, 505,281

Mauerhan, J. C., Muno, M. P., Morris, M. R., Stolovy, S. R., \& Cotera, A. 2010, ApJ, 710, 706

Mauerhan, J. C., van Dyk, S. D., \& Morris, P. W. 2009, PASP, 121, 591

McClintock, J. E., \& Remillard, R. A. 2006, in Compact Stellar X-ray Sources, ed. W. H. G. Lewin \& M. van der Klis (Cambridge: Cambridge Univ. Press), 157

Monet, D. G., et al. 2003, AJ, 125, 984
Morris, P. W., Eenens, P. R. J., Hanson, M. M., Conti, P. S., \& Blum, R. D. 1996, ApJ, 470, 597

Motch, C., et al. 2010, A\&A, 523, A92

Murphy, T., Mauch, T., Green, A., Hunstead, R. W., Piestrzynska, B., Kels, A. P., \& Sztajer, P. 2007, MNRAS, 382, 382

Murray, S. S. 2000, American Physical Society April Meeting, Abstract J8.003

Negueruela, I., Smith, D. M., Reig, P., Chaty, S., \& Torrejón, J. M. 2006, in The X-ray Universe 2005, ed. A. Wilson (ESA SP-604; Noordwijk: ESA), 165

Osip, D. J., Floyd, D., \& Covarrubias, R. 2008, Proc. SPIE, 7014, 70140A

Oskinova, L. M. 2005, MNRAS, 361, 679

Pestalozzi, M., Torkelsson, U., Hobbs, G., \& López-Sánchez, Á. R. 2009, A\&A, 506, L21

Pittard, J. M., \& Dougherty, S. M. 2006, MNRAS, 372, 801

Pittard, J. M., Dougherty, S. M., Coker, R. F., O’Connor, E., \& Bolingbroke, N. J. 2006, A\&A, 446, 1001

Pittard, J. M., \& Parkin, E. R. 2010, MNRAS, 403, 1657

Prilutskii, O. F., \& Usov, V. V. 1976, SvA, 20, 2

Raassen, A. J. J., van der Hucht, K. A., Mewe, R., Antokhin, I. I., Rauw, G., Vreux, J.-M., Schmutz, W., \& Güdel, M. 2003, A\&A, 402, 653

Raymond, J. C., \& Smith, B. W. 1977, ApJS, 35, 419

Reid, M. J., Menten, K. M., Zheng, X. W., Brunthaler, A., \& Xu, Y. 2009, ApJ, 705, 1548

Reig, P. 1999, A\&A, 345, 576

Reimer, A., Pohl, M., \& Reimer, O. 2006, ApJ, 644, 1118

Sana, H., Rauw, G., Nazé, Y., Gosset, E., \& Vreux, J. 2006, MNRAS, 372 , 661

Sguera, V., et al. 2006, ApJ, 646, 452

Shara, M. M., et al. 2009, AJ, 138, 402

Shaver, P. A., Retallack, D. S., Wamsteker, W., \& Danks, A. C. 1981, A\&A, 102,225

Skinner, S. L., Itoh, M., Nagase, F., \& Zhekov, S. A. 1999, ApJ, 524, 394

Skrutskie, M. F., et al. 2006, AJ, 131, 1163

Smith, L. F., Biermann, P., \& Mezger, P. G. 1978, A\&A, 66, 65

Smith, N., \& Conti, P. S. 2008, ApJ, 679, 1467

Stevens, I. R., Blondin, J. M., \& Pollock, A. M. T. 1992, ApJ, 386, 265

Sugizaki, M., Mitsuda, K., Kaneda, H., Matsuzaki, K., Yamauchi, S., \& Koyama, K. 2001, ApJS, 134, 77

Szostek, A., Zdziarski, A. A., \& McCollough, M. L. 2008, MNRAS, 388, 1001

Tam, P. H. T., Wagner, S. J., Tibolla, O., \& Chaves, R. C. G. 2010, A\&A, 518, A8

Tomsick, J. A., Chaty, S., Rodriguez, J., Walter, R., Kaaret, P., \& Tovmassian, G. 2009, ApJ, 694, 344

Usov, V. V. 1992, ApJ, 389, 635

van der Hucht, K. A. 2001, New Astron. Rev., 45, 135

van der Hucht, K. A. 2006, A\&A, 458, 453

Walborn, N. 2001, in ASP Conf. Ser. 242, Eta Carinae and Other Mysterious Stars, ed. T. R. Gull, S. Johannson, \& K. Davidson (San Francisco, CA: ASP), 217

Walborn, N. R. 1971, ApJS, 23, 257

Watson, C., et al. 2008, ApJ, 681, 1341

Watson, M. G., et al. 2009, A\&A, 493, 339

Zhekov, S. A., \& Park, S. 2010, ApJ, 709, L119 